\title{
Linear programming approach to matrix games with intuitionistic fuzzy goals
}

\author{
Jiang-Xia Nan \\ School of Mathematics and Computing Sciences \\ Guilin University of Electronic Technology \\ Guilin, Guangxi 541004, China \\ Deng-Feng $\mathbf{L i}{ }^{*}$ \\ School of Management, Fuzhou University \\ Fuzhou, Fujian 350108, China \\ E-mail: lidengfeng@fzu.edu.cn \\ Received 3 september 2010 \\ Accepted 28 September 2012
}

\begin{abstract}
Adding an additional degree of non-membership, K. T. Atanassov introduced the concept of the intuitionistic fuzzy (IF) set (IF-set), which has rarely been applied to the game theory yet. The aim of this paper is to develop the concept and methodology of matrix games with IF-set goals in which goals of players are expressed with IF-sets and payoffs are expressed with real numbers rather than IF-sets. In this methodology, the concepts of IF-set goals and the solutions of matrix games with IF-set goals are proposed. It is proven that solutions of matrix games with IF-set goals can be obtained through solving the developed auxiliary linear programming models, which are the generalization of matrix games with fuzzy goals. The proposed methodology is illustrated with a numerical example. Furthermore, comparison analysis of the proposed methodology is conducted to show its advantages over matrix games with fuzzy goals.
\end{abstract}

Keywords: Fuzzy game; Intuitionistic fuzzy set; Game theory; Linear programming; Soft computing

\section{Introduction}

Game theory has been used as a powerful analytical tool for decision making problems in the organizations or competitive systems. There are always uncertainty and imprecision existing in real-life decision making information. Therefore, taking into uncertainty and imprecision of information in decision making problems may be requested to construct mathematical models under fuzzy environments. Fuzzy game theory provides a way of solving real-life conflict problems with fuzzy information. Recently, an increasing number of papers and books ${ }^{1-15}$ have been published on this topic in which several types of fuzzy games have been investigated. The fuzzy set (F-set) uses only a membership function, which assigns to each element $x$ of the universe of discourse a number $\mu(x)$ from the unit interval $[0,1]$ to indicate the degree of belongingness to the F-set under consideration. The degree of non-belongingness is just automatically equal to $1-\mu(x)$. However, a human being who expresses the degree of membership of a given element in a F-set very often does not express corresponding degree of non-membership as the compliment to 1 . On the other hand, sometimes it seems to be more natural to describe imprecise and uncertain opinions not only by the degree of membership. It is due to the fact that in some situations players also describe their negative feelings, i.e., their degrees of dissatisfaction about the outcomes of the game. Furthermore, it is possible that players are not so sure about the outcomes of the game. In other words, players may have some degree of hesitation or uncertainty. Thus, Atanassov ${ }^{16-18}$ introduced the concept of the intuitionistic fuzzy (IF) set (IF-set) which is

\footnotetext{
* Corresponding author's address: School of Management, Fuzhou University, No. 2, Xueyuan Road, Daxue New District, Fuzhou District, Fuzhou, Fujian 350108, China. Tel./Fax: +86-0591-83768427.
} 
characterized by two functions expressing the degree of membership and the degree of non-membership, respectively. The degree of hesitation is equal to 1 minus both the degree of membership and the degree of non-membership. The IF-set has been applied to different areas $^{19-22}$. The idea of using the IF-set to represent the uncertain information of matrix games is useful. The reason is that the IF-set may indicate players' preference information in terms of favor, against and neutral. Dealing with uncertain information, the IF-set may indicate information more abundant and flexible than the F-set. However, there exists less investigation on matrix games using the IF-set. Atanassov $^{23}$ firstly described a game problem using the IF-set. Dimitrov ${ }^{24,} 25$ applied the IF-set to some market structure problems from a view point of economic orientation. Tenekedjiev et $\mathrm{al}^{26}$ discussed the rational conservative betting on sport game events by a fuzzy (partially rational) decision maker with the help of generalized lotteries of II type. The scheme accounted for the interval-valued character of probability elicitation results which were equivalently described by the IF-set in the mathematical expression. The fuzzy rational lottery with IF-set representation of the state uncertainty was proposed. Classical and conservative methods were proposed to transform the fuzzy rational lottery into ordinary lotteries, which were solved by the ordinary methods such as Wald's maximin principle and utility theory. $\mathrm{Li}$ and $\mathrm{Nan}^{27}$ studied the matrix games in which payoffs of players are expressed with the IF-set and goals of players are not taken into consideration. The concepts of the solutions for matrix games with payoffs of IF-sets were defined and hereby a method was developed on the basis of a pair of auxiliary nonlinear/linear programming models, which were derived from the constructed bi-objective programming models. $\mathrm{Li}^{28}$ further extended the method ${ }^{27}$ to study the matrix games with payoffs of interval-valued IF-sets. The discussed matrix games ${ }^{27,} 28$ only taken into consideration uncertainty in payoffs of players, which was expressed with IF-sets. In reality, however, players may have their expected goals for the outcome of the game. The goals may be given by players with some uncertainty. Namely, players may have IF-set goals which are expressed with IF-sets (please see the following Definition 2). Therefore, game with IF-set goals is an important type of games in game theory. However, there exists no investigation on games with
IF-set goals. In this paper, we study matrix games with IF-set goals in which goals of players are expressed with IF-sets and payoffs of players are real numbers rather than IF-sets. It is not difficult to see that the matrix game with IF-set goals differs from the matrix game with F-set goals, which was studied by Sakawa and Nishizaki ${ }^{12,13}$ and Bector et al., 2 The former uses the degree of membership and the degree of nonmembership to express players' goals while the latter only uses the degree of membership to express players' goals. Thus, the degree of hesitation of the IF-set goals may not be equal to 0 while the degree of hesitation of the F-set goals is always equal to 0 .

The rest of this paper is organized as follows. The concept of an IF-set is briefly introduced in Section 2. In Section 3, matrix games with IF-set goals are formulated and the concept of their solutions is defined. It is proven that the solutions of matrix games with IFset goals can be obtained through solving the newly auxiliary linear programming models derived from a pair of bi-objective programming models. A numerical example and conclusion are given in Sections 4 and 5, respectively.

\section{Intuitionistic Fuzzy Sets}

Definition 1. (Atanassov ${ }^{16-18}$ ) Let $Z=\left\{z_{1}, z_{2}, \cdots, z_{n}\right\}$ be a finite universal set. An IF-set $C$ in $Z$ is an object having the form as $C=\left\{<z_{l}, \mu_{C}\left(z_{l}\right), v_{C}\left(z_{l}\right)>\mid z_{l} \in Z\right\}$, where $\mu_{C}\left(z_{l}\right) \in[0,1]$ and $v_{C}\left(z_{l}\right) \in[0,1]$ are the degree of membership and degree of non-membership of an element $z_{l} \in Z$ to the set $C \subseteq Z$, respectively, and such that for every $z_{l} \in Z$, they satisfy the condition: $0 \leq \mu_{C}\left(z_{l}\right)+v_{C}\left(z_{l}\right) \leq 1$.

Let $\pi_{C}\left(z_{l}\right)=1-\mu_{C}\left(z_{l}\right)-v_{C}\left(z_{l}\right)$, which is called the intuitionistic index of an element $z_{l}$ in the set $C$. It is the degree of indeterminacy membership of the element $z_{l}$ to the set $C$. Obviously, $0 \leq \pi_{C}\left(z_{l}\right) \leq 1$.

\section{IF-set goals and solving methods for matrix games with IF-set goals}

Let us consider the matrix games in which goals are expressed with IF-sets and payoffs of players are expressed with real numbers rather than IF-sets. For conciseness, such matrix games are called as matrix games with IF-set goals in the sequent. Assume that $S_{1}=\left\{\delta_{1}, \delta_{2}, \cdots, \delta_{m}\right\}$ and $S_{2}=\left\{\sigma_{1}, \sigma_{2}, \cdots, \sigma_{n}\right\}$ are sets of pure strategies for two players I and II, respectively. 
The vectors $\boldsymbol{x}=\left(x_{1}, x_{2}, \cdots, x_{m}\right)^{\mathrm{T}}$ and $\boldsymbol{y}=\left(y_{1}, y_{2}, \cdots, y_{n}\right)^{\mathrm{T}}$ are mixed strategies for players I and II, respectively, where $x_{i} \quad(i=1,2, \cdots, m)$ and $y_{j} \quad(j=1,2, \cdots, n)$ are probabilities in which players I and II choose their pure strategies $\delta_{i} \in S_{1}$ and $\sigma_{j} \in S_{2}$, respectively. Sets of all mixed strategies for players I and II are denoted by $X$ and $Y$, respectively, i.e., $X=\left\{\boldsymbol{x} \mid \sum_{i=1}^{m} x_{i}=1, x_{i} \geq 0(i=1,2, \cdots, m)\right\}$ and $Y=\left\{\boldsymbol{y} \mid \sum_{j=1}^{n} y_{j}=1, y_{j} \geq 0(j=1,2, \cdots, n)\right\}$. The payoff matrix for player $\mathrm{I}$ is concisely expressed as the matrix $\boldsymbol{F}=\left(a_{i j}\right)_{m \times n}$, where every $a_{i j}(i=1,2, \cdots, m ; j=1,2, \cdots, n)$ is a real number. The payoff matrix for player II is $\boldsymbol{- F}=\left(-a_{i j}\right)_{m \times n}$ since the matrix game is zero-sum.

In reality, however, players may have their respective expected goals for the outcome of the game. Generally, players may give their goals according to their judgments and estimations. However, these judgments and estimations are uncertain and imprecise or vague. In order to accommodate uncertain and imprecise nature of human judgment and estimation, we employ the IF-set to describe each player' goal, i.e., assume that each player has an IF-set goal, which is defined as follows.

Definition 2. (IF-set goals) Denote the set of the expected payoff of player $I$ by $D=\left\{\boldsymbol{x}^{\mathrm{T}} \boldsymbol{F} \boldsymbol{y} \mid(\boldsymbol{x}, \boldsymbol{y}) \in X \times Y\right\} \subseteq \mathrm{R}$, where $\mathrm{R}$ is the set of real numbers. Then, an IF-set goal $A=\left\{<\boldsymbol{x}^{\mathrm{T}} \boldsymbol{F} \boldsymbol{y}, \mu_{A}\left(\boldsymbol{x}^{\mathrm{T}} \boldsymbol{F} \boldsymbol{y}\right), v_{A}\left(\boldsymbol{x}^{\mathrm{T}} \boldsymbol{F} \boldsymbol{y}\right)>\mid \boldsymbol{x}^{\mathrm{T}} \boldsymbol{F} \boldsymbol{y} \in D\right\} \quad$ with respect to the payoff for player $I$ is an IF-set on the set $D$, whose membership and non-membership functions are defined as follows:

$$
\begin{gathered}
\mu_{A}: D \rightarrow[0,1] \\
\boldsymbol{x}^{\mathrm{T}} \boldsymbol{F} \boldsymbol{y} \in D \mapsto \mu_{A}\left(\boldsymbol{x}^{\mathrm{T}} \boldsymbol{F} \boldsymbol{y}\right)
\end{gathered}
$$

and

$$
\begin{gathered}
v_{A}: D \rightarrow[0,1] \\
\boldsymbol{x}^{\mathrm{T}} \boldsymbol{F} \boldsymbol{y} \in D \mapsto v_{A}\left(\boldsymbol{x}^{\mathrm{T}} \boldsymbol{F} \boldsymbol{y}\right),
\end{gathered}
$$

which satisfy the condition: $0 \leq \mu_{A}\left(\boldsymbol{x}^{\mathrm{T}} \boldsymbol{F} \boldsymbol{y}\right)+v_{A}\left(\boldsymbol{x}^{\mathrm{T}} \boldsymbol{F} \boldsymbol{y}\right) \leq 1$.

$$
\text { Similarly, an IF-set goal }
$$$$
B=\left\{<\boldsymbol{x}^{\mathrm{T}} \boldsymbol{F} \boldsymbol{y}, \mu_{B}\left(\boldsymbol{x}^{\mathrm{T}} \boldsymbol{F} \boldsymbol{y}\right), v_{B}\left(\boldsymbol{x}^{\mathrm{T}} \boldsymbol{F} \boldsymbol{y}\right)>\mid \boldsymbol{x}^{\mathrm{T}} \boldsymbol{F} \boldsymbol{y} \in D\right\} \quad \text { with }
$$
respect to the payoff for player II is an IF-set on the set $D$, whose membership and non-membership functions are defined as follows:

$$
\begin{gathered}
\mu_{B}: D \rightarrow[0,1] \\
\boldsymbol{x}^{\mathrm{T}} \boldsymbol{F} \boldsymbol{y} \in D \mapsto \mu_{B}\left(\boldsymbol{x}^{\mathrm{T}} \boldsymbol{F} \boldsymbol{y}\right) \in[0,1]
\end{gathered}
$$

and

$$
\begin{gathered}
v_{B}: D \rightarrow[0,1] \\
\boldsymbol{x}^{\mathrm{T}} \boldsymbol{F} \boldsymbol{y} \in D \mapsto v_{B}\left(\boldsymbol{x}^{\mathrm{T}} \boldsymbol{F y}\right) \in[0,1],
\end{gathered}
$$

which satisfy the condition: $0 \leq \mu_{B}\left(\boldsymbol{x}^{\mathrm{T}} \boldsymbol{F} \boldsymbol{y}\right)+v_{B}\left(\boldsymbol{x}^{\mathrm{T}} \boldsymbol{F} \boldsymbol{y}\right) \leq 1$.
Obviously, if $\mu_{A}\left(\boldsymbol{x}^{\mathrm{T}} \boldsymbol{F} \boldsymbol{y}\right)+v_{A}\left(\boldsymbol{x}^{\mathrm{T}} \boldsymbol{F y}\right)=1$ then the IF-set goal $A$ for player $\mathrm{I}$ is reduced to the $\mathrm{F}$-set goal $A^{\prime}=\left\{\boldsymbol{x}^{\mathrm{T}} \boldsymbol{F} \boldsymbol{y}, \mu_{A}\left(\boldsymbol{x}^{\mathrm{T}} \boldsymbol{F} \boldsymbol{y}\right), 1-\mu_{A}\left(\boldsymbol{x}^{\mathrm{T}} \boldsymbol{F} \boldsymbol{y}\right)>\mid \boldsymbol{x}^{\mathrm{T}} \boldsymbol{F} \boldsymbol{y} \in D\right\}$.

Similarly, if $\mu_{B}\left(\boldsymbol{x}^{\mathrm{T}} \boldsymbol{F} \boldsymbol{y}\right)+v_{B}\left(\boldsymbol{x}^{\mathrm{T}} \boldsymbol{F} \boldsymbol{y}\right)=1$ then the IF-set goal $B$ for player II is reduces to the F-set goal $B^{\prime}=\left\{<\boldsymbol{x}^{\mathrm{T}} \boldsymbol{F} \boldsymbol{y}, \mu_{B}\left(\boldsymbol{x}^{\mathrm{T}} \boldsymbol{F} \boldsymbol{y}\right), 1-\mu_{B}\left(\boldsymbol{x}^{\mathrm{T}} \boldsymbol{F} \boldsymbol{y}\right)>\mid \boldsymbol{x}^{\mathrm{T}} \boldsymbol{F} \boldsymbol{y} \in D\right\}$.

The concept of the IF-set goal is a generalization of that introduced by Sakawa and Nishizaki ${ }^{12,13}$ and Bector et al. ${ }^{1,2}$ in that the former adds an additional nonmembership function and hereby may describe the hesitancy of players' judgment and estimation.

The degree of membership $\mu_{A}\left(\boldsymbol{x}^{\mathrm{T}} \boldsymbol{F} \boldsymbol{y}\right)$ for the player I's IF-set goal $A$ can be interpreted as the degree of satisfaction for an expected payoff. The degree of nonmembership $v_{A}\left(\boldsymbol{x}^{\mathrm{T}} \boldsymbol{F y}\right)$ for the player I's IF-set goal $A$ can be interpreted as the degree of dissatisfaction for an expected payoff. Similarly, $\mu_{B}\left(\boldsymbol{x}^{\mathrm{T}} \boldsymbol{F y}\right)$ and $v_{B}\left(\boldsymbol{x}^{\mathrm{T}} \boldsymbol{F y}\right)$ can be interpreted as the satisfaction and dissatisfaction degrees of player II for an expected payoff.

An IF-set goal is characterized by a pair of membership and non-membership functions, which map a domain of payoffs into the range of the degree of satisfaction and the range of the degree of dissatisfaction of attainment for the IF-set goal, respectively. Assume that player I may prefer the payoff possessing the higher membership degree and the lower non-membership degree. Namely, player I wants to maximize the degree of satisfaction of attainment of the IF-set goal and minimize the degree of dissatisfaction of attainment of the IF-set goal. Whereas player II will choose a strategy so as to minimize player I's degree of satisfaction and maximize player I's degree of dissatisfaction of attainment of the IF-set goal. Thus, the concept of a maximin-minimax solution for matrix games with IF-set goals is given as follows.

Definition 3. Let $\mu_{A}\left(x^{\mathrm{T}} \mathrm{Fy}\right)$ and $v_{A}\left(x^{\mathrm{T}} \mathbf{F y}\right)$ be the degrees of membership and non-membership of the IFset goal for player I when I and II choose strategies $\boldsymbol{x}$ and $\boldsymbol{y}$, respectively. Then, player I's maximin-minimax value with respect to the attainment degree of the IF-set goal is defined as follows:

$$
\left\{\begin{array}{l}
\max _{\boldsymbol{x} \in X} \min _{\boldsymbol{y} \in Y}\left\{\mu_{A}\left(\boldsymbol{x}^{\mathrm{T}} \boldsymbol{F} \boldsymbol{y}\right)\right\} \\
\min _{\boldsymbol{x} \in X} \max _{\boldsymbol{y} \in Y}\left\{v_{A}\left(\boldsymbol{x}^{\mathrm{T}} \boldsymbol{F} \boldsymbol{y}\right)\right\}
\end{array}\right.
$$

Such a strategy $\boldsymbol{x}^{*}$ is called the maximin-minimax solution of matrix games with the IF-set goals for I.

Similarly, player II's maximin-minimax value with respect to the attainment degree of the IF-set goal is defined as follows: 


$$
\left\{\begin{array}{c}
\max _{\boldsymbol{y} \in Y} \min _{\boldsymbol{x} \in X}\left\{\mu_{B}\left(\boldsymbol{x}^{\mathrm{T}} \boldsymbol{F} \boldsymbol{y}\right)\right\} \\
\min _{\boldsymbol{y} \in Y} \max _{\boldsymbol{x} \in X}\left\{v_{B}\left(\boldsymbol{x}^{\mathrm{T}} \boldsymbol{F} \boldsymbol{y}\right)\right\}
\end{array}\right.
$$

Such a strategy $y^{*}$ is called the maximin-minimax solution of matrix games with the IF-set goals for II.

Obviously, the concept of the solution of matrix games with IF-set goals is different from that proposed by Sakawa and Nishizaki ${ }^{12,13}$ and Bector et al. ${ }^{1,2}$ in that the former has the additional non-membership function and hereby needs to optimize both the membership function and the non-membership function simultaneously. Moreover, the concept of the solution of matrix games with the IF-set goals is different from that proposed by $\mathrm{Li}$ and $\mathrm{Nan}^{27}$ and $\mathrm{Li}^{28}$ in that the latter took into consideration the IF-set payoffs rather than the IF-set goals.

In the sequent, optimization models are constructed for players I and II so as to obtain the maximin-minimax solutions of the matrix games with IF-set goals.

The membership function $\mu_{A}$ and non-membership function $v_{A}$ of the IF-set goal for player I may be of different forms according to the real situations and needs. Here, we choose the linear functions of membership and non-membership as follows:

$$
\mu_{A}\left(\boldsymbol{x}^{\mathrm{T}} \boldsymbol{F} \boldsymbol{y}\right)=\left\{\begin{array}{cc}
0 & \text { if } \boldsymbol{x}^{\mathrm{T}} \boldsymbol{F} \boldsymbol{y}<v_{a}-p_{a} \\
1-\left(v_{a}-\boldsymbol{x}^{\mathrm{T}} \boldsymbol{F y}\right) / p_{a} & \text { if } v_{a}-p_{a} \leq \boldsymbol{x}^{\mathrm{T}} \boldsymbol{F} \boldsymbol{y}<v_{a} \\
1 & \text { if } \boldsymbol{x}^{\mathrm{T}} \boldsymbol{F} \boldsymbol{y} \geq v_{a}
\end{array}\right.
$$

and

$$
v_{A}\left(\boldsymbol{x}^{\mathrm{T}} \boldsymbol{F y}\right)=\left\{\begin{array}{cc}
1 & \text { if } \boldsymbol{x}^{\mathrm{T}} \boldsymbol{F} \boldsymbol{y}<v_{r}-p_{r} \\
\left(v_{r}-\boldsymbol{x}^{\mathrm{T}} \boldsymbol{F}\right) / p_{r} & \text { if } v_{r}-p_{r} \leq \boldsymbol{x}^{\mathrm{T}} \boldsymbol{F} \boldsymbol{y}<v_{r} \\
0 & \text { if } \boldsymbol{x}^{\mathrm{T}} \boldsymbol{F} \boldsymbol{y} \geq v_{r}
\end{array}\right.
$$

respectively, depicted in Fig.1, where $v_{a}$ and $p_{a}$ are the aspiration level and the corresponding tolerance error for player I, $v_{r}$ and $p_{r}$ are the rejection level and the corresponding tolerance error for player I, $\omega_{a}$ and $q_{a}$ are the aspiration level and the corresponding tolerance error for player II, $\omega_{r}$ and $q_{r}$ are the rejection level and the corresponding tolerance error for player II, and $v_{r}-p_{r} \leq v_{a}-p_{a}$ and $v_{r} \leq v_{a}$.

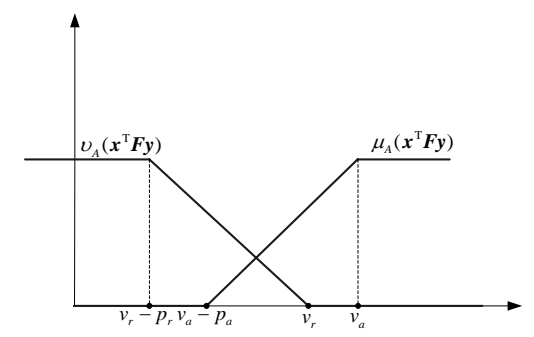

Fig.1. Player I's IF-set goal $<\mu_{A}\left(\boldsymbol{x}^{\mathrm{T}} \boldsymbol{F} \boldsymbol{y}\right), v_{A}\left(\boldsymbol{x}^{\mathrm{T}} \boldsymbol{F} \boldsymbol{y}\right)>$
The following conclusion is easily reached from Eqs. (3) and (4).

Theorem 1. Let $\mu_{A}\left(x^{\mathrm{T}} \mathbf{F y}\right)$ and $v_{A}\left(x^{\mathrm{T}} \mathbf{F y}\right)$ be the degree of membership and the degree of nonmembership, which are defined by Eqs. (3) and (4), respectively.

Then $A=\left\{<\boldsymbol{x}^{\mathrm{T}} \boldsymbol{F} \boldsymbol{y}, \mu_{A}\left(\boldsymbol{x}^{\mathrm{T}} \boldsymbol{F} \boldsymbol{y}\right), v_{A}\left(\boldsymbol{x}^{\mathrm{T}} \boldsymbol{F} \boldsymbol{y}\right)>\mid \boldsymbol{x}^{\mathrm{T}} \boldsymbol{F} \boldsymbol{y} \in D\right\}$ is an IFset.

Proof. It is easily derived from Eqs. (3) and (4) that

$$
0 \leq \mu_{A}\left(\boldsymbol{x}^{\mathrm{T}} \boldsymbol{F} \boldsymbol{y}\right) \leq 1
$$

and

$$
0 \leq v_{A}\left(\boldsymbol{x}^{\mathrm{T}} \boldsymbol{F} \boldsymbol{y}\right) \leq 1 .
$$

(a) If $\boldsymbol{x}^{\mathrm{T}} \boldsymbol{F} \boldsymbol{y} \leq v_{a}-p_{a}$ then it is easily seen from Eqs. (3) and (4) that $0 \leq \mu_{A}\left(\boldsymbol{x}^{\mathrm{T}} \boldsymbol{F} \boldsymbol{y}\right)+v_{A}\left(\boldsymbol{x}^{\mathrm{T}} \boldsymbol{F} \boldsymbol{y}\right) \leq 1$.

(b) If $\boldsymbol{x}^{\mathrm{T}} \boldsymbol{F} \boldsymbol{y} \geq v_{r}$ then it is easily seen from Eqs. (3) and (4) that $0 \leq \mu_{A}\left(\boldsymbol{x}^{\mathrm{T}} \boldsymbol{F} \boldsymbol{y}\right)+v_{A}\left(\boldsymbol{x}^{\mathrm{T}} \boldsymbol{F} \boldsymbol{y}\right) \leq 1$.

(c) If $v_{a}-p_{a} \leq \boldsymbol{x}^{\mathrm{T}} \boldsymbol{F} \boldsymbol{y} \leq v_{r}$ then it follows from Eqs. (3) and (4) that

$$
\begin{aligned}
\mu_{A}\left(\boldsymbol{x}^{\mathrm{T}} \boldsymbol{F} \boldsymbol{y}\right)+v_{A}\left(\boldsymbol{x}^{\mathrm{T}} \boldsymbol{F} \boldsymbol{y}\right) & =1-\frac{v_{a}-\boldsymbol{x}^{\mathrm{T}} \boldsymbol{F} \boldsymbol{y}}{p_{a}}+\frac{v_{r}-\boldsymbol{x}^{\mathrm{T}} \boldsymbol{F} \boldsymbol{y}}{p_{r}} \\
= & \frac{p_{a}-v_{a}+\boldsymbol{x}^{\mathrm{T}} \boldsymbol{F} \boldsymbol{y}}{p_{a}}+\frac{v_{r}-\boldsymbol{x}^{\mathrm{T}} \boldsymbol{F} \boldsymbol{y}}{p_{r}} .
\end{aligned}
$$

Hence,

$$
\mu_{A}\left(\boldsymbol{x}^{\mathrm{T}} \boldsymbol{F} \boldsymbol{y}\right)+v_{A}\left(\boldsymbol{x}^{\mathrm{T}} \boldsymbol{F} \boldsymbol{y}\right) \geq 0
$$

since $\boldsymbol{x}^{\mathrm{T}} \boldsymbol{F} \boldsymbol{y}+p_{a}-v_{a} \geq 0, \quad v_{r}-\boldsymbol{x}^{\mathrm{T}} \boldsymbol{F} \boldsymbol{y} \geq 0, \quad p_{a} \geq 0$ and $p_{r} \geq 0$.

On the other hand, if $v_{a}-p_{a} \leq \boldsymbol{x}^{\mathrm{T}} \boldsymbol{F} \boldsymbol{y} \leq v_{r}$ and $p_{r}-p_{a}<0$ then due to $-v_{a}+p_{a} \leq-v_{r}+p_{r}$, we have

$$
\begin{gathered}
\mu_{A}\left(\boldsymbol{x}^{\mathrm{T}} \boldsymbol{F} \boldsymbol{y}\right)+v_{A}\left(\boldsymbol{x}^{\mathrm{T}} \boldsymbol{F} \boldsymbol{y}\right)=1-\frac{v_{a}-\boldsymbol{x}^{\mathrm{T}} \boldsymbol{F} \boldsymbol{y}}{p_{a}}+\frac{v_{r}-\boldsymbol{x}^{\mathrm{T}} \boldsymbol{F} \boldsymbol{y}}{p_{r}} \\
=\frac{p_{a} p_{r}+\boldsymbol{x}^{\mathrm{T}} \boldsymbol{F} \boldsymbol{y}\left(p_{r}-p_{a}\right)-p_{r} v_{a}+p_{a} v_{r}}{p_{a} p_{r}} \\
\leq \frac{p_{a} p_{r}+\left(v_{a}-p_{a}\right)\left(p_{r}-p_{a}\right)-p_{r} v_{a}+p_{a} v_{r}}{p_{a} p_{r}} \\
=\frac{\left(-v_{a}+p_{a}\right)+v_{r}}{p_{r}} \leq \frac{\left(-v_{r}+p_{r}\right)+v_{r}}{p_{r}}=1
\end{gathered}
$$

If $v_{a}-p_{a} \leq \boldsymbol{x}^{\mathrm{T}} \boldsymbol{F} \boldsymbol{y} \leq v_{r}$ and $p_{r}-p_{a}>0$, then

$$
\begin{aligned}
& \mu_{A}\left(\boldsymbol{x}^{\mathrm{T}} \boldsymbol{F} \boldsymbol{y}\right)+v_{A}\left(\boldsymbol{x}^{\mathrm{T}} \boldsymbol{F} \boldsymbol{y}\right) \\
= & 1-\frac{v_{a}-\boldsymbol{x}^{\mathrm{T}} \boldsymbol{F} \boldsymbol{y}}{p_{a}}+\frac{v_{r}-\boldsymbol{x}^{\mathrm{T}} \boldsymbol{F} \boldsymbol{y}}{p_{r}} \\
= & \frac{p_{a} p_{r}+\boldsymbol{x}^{\mathrm{T}} \boldsymbol{F} \boldsymbol{y}\left(p_{r}-p_{a}\right)-p_{r} v_{a}+p_{a} v_{r}}{p_{a} p_{r}}
\end{aligned}
$$




$$
\begin{aligned}
& \leq \frac{p_{a} p_{r}+v_{r}\left(p_{r}-p_{a}\right)-p_{r} v_{a}+p_{a} v_{r}}{p_{a} p_{r}} \\
& =\frac{p_{a} p_{r}+v_{r} p_{r}-v_{r} p_{a}-p_{r} v_{a}+p_{a} v_{r}}{p_{a} p_{r}} \\
& \leq \frac{p_{a}+v_{r}-v_{a}}{p_{a}} \leq \frac{p_{a}+v_{a}-v_{a}}{p_{a}}=1
\end{aligned}
$$

since $v_{r} \leq v_{a}$.

$$
\text { If } v_{a}-p_{a} \leq \boldsymbol{x}^{\mathrm{T}} \boldsymbol{F} \boldsymbol{y} \leq v_{r} \text { and } p_{r}-p_{a}=0 \text {, i.e., }
$$

$p_{r}=p_{a}$, then due to $v_{r} \leq v_{a}$, we have

$$
\begin{aligned}
& \mu_{A}\left(\boldsymbol{x}^{\mathrm{T}} \boldsymbol{F} \boldsymbol{y}\right)+v_{A}\left(\boldsymbol{x}^{\mathrm{T}} \boldsymbol{F} \boldsymbol{y}\right)=1-\frac{v_{a}-\boldsymbol{x}^{\mathrm{T}} \boldsymbol{F} \boldsymbol{y}}{p_{a}}+\frac{v_{r}-\boldsymbol{x}^{\mathrm{T}} \boldsymbol{F} \boldsymbol{y}}{p_{r}} \\
= & \frac{p_{a} p_{r}+\boldsymbol{x}^{\mathrm{T}} \boldsymbol{F} \boldsymbol{y}\left(p_{r}-p_{a}\right)-p_{r} v_{a}+p_{a} v_{r}}{p_{a} p_{r}} \\
= & \frac{p_{a} p_{r}-p_{r} v_{a}+p_{a} v_{r}}{p_{a} p_{r}} \\
= & 1-\frac{p_{r} v_{a}-p_{a} v_{r}}{p_{a} p_{r}}=1-\frac{v_{a}-v_{r}}{p_{a}} \leq 1
\end{aligned}
$$

Combining with Eqs. (5)-(8), we have: $0 \leq \mu_{A}\left(\boldsymbol{x}^{\mathrm{T}} \boldsymbol{F} \boldsymbol{y}\right)+v_{A}\left(\boldsymbol{x}^{\mathrm{T}} \boldsymbol{F} \boldsymbol{y}\right) \leq 1$. Summarizing the cases (a)(c), we have proven that $A$ is an IF-set.

The IF-set goal $A$ means that player I is not satisfied with an expected payoff $\boldsymbol{x}^{\mathrm{T}} \boldsymbol{F} \boldsymbol{y}$ being smaller than $v_{a}-p_{a}$, whereas I may not completely reject if $\boldsymbol{x}^{\mathrm{T}} \boldsymbol{F} \boldsymbol{y}$ is smaller than $v_{a}-p_{a}$. Player I may designate $v_{r}-p_{r}$ as a rejection level, i.e., player I may think that $\boldsymbol{x}^{\mathrm{T}} \boldsymbol{F} \boldsymbol{y}$ being smaller than $v_{r}-p_{r}$ cannot be accepted. The degree of satisfaction increases linearly as an expected payoff $\boldsymbol{x}^{\mathrm{T}} \boldsymbol{F} \boldsymbol{y}$ becomes larger than $v_{a}-p_{a}$. On the other hand, the degree of dissatisfaction decreases linearly as an expected payoff $\boldsymbol{x}^{\mathrm{T}} \boldsymbol{F} \boldsymbol{y}$ becomes larger than $v_{r}-p_{r}$ and is zero till $\boldsymbol{x}^{\mathrm{T}} \boldsymbol{F y}$ is $v_{r}$. But player I may not completely accept if $\boldsymbol{x}^{\mathrm{T}} \boldsymbol{F y}$ is larger than $v_{r}$ and player I satisfies enough with an expected payoff $\boldsymbol{x}^{\mathrm{T}} \boldsymbol{F} \boldsymbol{y}$ being larger than $v_{a}$. The gap between $v_{r}-p_{r}$ and $v_{a}$ needs to be determined, i.e., player I always has the degree of hesitation. The length of the uncertainty is $v_{a}-v_{r}+p_{r}$.

Likewise, the membership function $\mu_{B}\left(\boldsymbol{x}^{\mathrm{T}} \boldsymbol{F} \boldsymbol{y}\right)$ and the non-membership function $v_{B}\left(\boldsymbol{x}^{\mathrm{T}} \boldsymbol{F y}\right)$ of the IF-set goal for player II are chosen to be linear, i.e.,

$$
\mu_{B}\left(\boldsymbol{x}^{\mathrm{T}} \boldsymbol{F} \boldsymbol{y}\right)=\left\{\begin{array}{cc}
1 & \text { if } \boldsymbol{x}^{\mathrm{T}} \boldsymbol{F} \boldsymbol{y}<\omega_{a} \\
1-\left(\boldsymbol{x}^{\mathrm{T}} \boldsymbol{F} \boldsymbol{y}-\omega_{a}\right) / q_{a} & \text { if } \omega_{a} \leq \boldsymbol{x}^{\mathrm{T}} \boldsymbol{F} \boldsymbol{y}<\omega_{a}+q_{a} \\
0 & \text { if } \boldsymbol{x}^{\mathrm{T}} \boldsymbol{F} \boldsymbol{y} \geq \omega_{a}+q_{a}
\end{array}\right.
$$

and

$$
v_{B}\left(\boldsymbol{x}^{\mathrm{T}} \boldsymbol{F} \boldsymbol{y}\right)=\left\{\begin{array}{cc}
0 & \text { if } \boldsymbol{x}^{\mathrm{T}} \boldsymbol{F} \boldsymbol{y}<\omega_{r} \\
\left(\boldsymbol{x}^{\mathrm{T}} \boldsymbol{F} \boldsymbol{y}-\omega_{r}\right) / q_{r} & \text { if } \omega_{r} \leq \boldsymbol{x}^{\mathrm{T}} \boldsymbol{F} \boldsymbol{y}<\omega_{r}+q_{r} \\
1 & \text { if } \boldsymbol{x}^{\mathrm{T}} \boldsymbol{F} \boldsymbol{y} \geq \omega_{r}+q_{r}
\end{array}\right.
$$

respectively, where $\omega_{a} \leq \omega_{r}$ and $\omega_{a}+q_{a} \leq \omega_{r}+q_{r}$, depicted in Fig. 2.

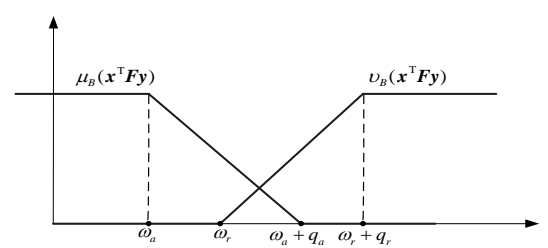

Fig. 2. Player II's IF-set goal $<\mu_{B}\left(\boldsymbol{x}^{\mathrm{T}} \boldsymbol{F} \boldsymbol{y}\right), v_{B}\left(\boldsymbol{x}^{\mathrm{T}} \boldsymbol{F} \boldsymbol{y}\right)>$

Similarly, $B$ can be proven to be an IF-set.

The IF-set goal $B$ means that player II satisfies an expected payoff $\boldsymbol{x}^{\mathrm{T}} \boldsymbol{F} \boldsymbol{y}$ being smaller than $\omega_{r}$. But II may not completely accept if $\boldsymbol{x}^{\mathrm{T}} \boldsymbol{F} \boldsymbol{y}$ is smaller than $\omega_{r}$. Player II may satisfy enough an expected payoff $\boldsymbol{x}^{\mathrm{T}} \boldsymbol{F} \boldsymbol{y}$ being smaller than $\omega_{a}$. Player II's degree of satisfaction decreases linearly as the expected payoff $\boldsymbol{x}^{\mathrm{T}} \boldsymbol{F} \boldsymbol{y}$ becomes larger than $\omega_{a}$ and is zero till $\boldsymbol{x}^{\mathrm{T}} \boldsymbol{F} \boldsymbol{y}$ is $\omega_{a}+q_{a}$. But at the same time, II may not completely reject if $\boldsymbol{x}^{\mathrm{T}} \boldsymbol{F} \boldsymbol{y}$ is larger than $\omega_{a}+q_{a}$. Player II may designate $\omega_{r}+q_{r}$ as rejection level, i.e., II may completely reject if the expected payoff $\boldsymbol{x}^{\mathrm{T}} \boldsymbol{F} \boldsymbol{y}$ is larger than $\omega_{r}+q_{r}$. The degree of dissatisfaction decreases linearly as an expected payoff $\boldsymbol{x}^{\mathrm{T}} \boldsymbol{F} \boldsymbol{y}$ becomes smaller than $\omega_{r}+q_{r}$. The gap between $\omega_{a}$ and $\omega_{r}+q_{r}$ needs to be determined, i.e., II always has the degree of hesitation. The length of the uncertainty is $\omega_{r}+q_{r}-\omega_{a}$.

Theorem 2. Assume that $\mu_{A}$ and $v_{A}$ for player I's IFset goal are the linear functions defined by Eqs. (3) and (4), respectively. Player I's maximin-minimax solution of the matrix game with the IF-set goal can be obtained through solving the linear programming as follows:

$$
\begin{aligned}
& \max \{\alpha-\beta\} \\
& \text { s.t. }\left\{\begin{array}{l}
\sum_{i=1}^{m} a_{i j} x_{i}+p_{a}-v_{a} \geq p_{a} \alpha \quad(j=1,2, \cdots n) \\
\sum_{i=1}^{m} a_{i j} x_{i}-v_{r} \geq-p_{r} \beta \quad(j=1,2, \cdots n) \\
x_{1}+x_{2}+\cdots+x_{m}=1 \\
0 \leq \alpha \leq 1,0 \leq \beta \leq 1 \\
\alpha+\beta \leq 1 \\
x_{i} \geq 0(i=1,2, \cdots, m)
\end{array}\right.
\end{aligned}
$$


Proof. According to Eqs. (3) and (4), Eq. (1) are calculated as follows:

$$
\begin{aligned}
\max _{\boldsymbol{x} \in X} \min _{\boldsymbol{y} \in Y}\left\{\mu_{\mathrm{A}}\left(\boldsymbol{x}^{\mathrm{T}} \boldsymbol{F} \boldsymbol{y}\right)\right\}=\max _{\boldsymbol{x} \in X} \min _{\boldsymbol{y} \in Y}\left\{1-\frac{v_{a}-\boldsymbol{x}^{\mathrm{T}} \boldsymbol{F} \boldsymbol{y}}{p_{a}}\right\} \\
=\frac{1}{p_{a}} \max _{\boldsymbol{x} \in X} \min _{\boldsymbol{y} \in Y}\left\{\sum_{i=1}^{m} \sum_{j=1}^{n} a_{i j} x_{i} y_{j}+p_{a}-v_{a}\right\} \\
=\frac{1}{p_{a}} \max _{x \in X} \min _{\boldsymbol{y} \in Y}\left\{\sum_{i=1}^{m} \sum_{j=1}^{n} a_{i j} x_{i} y_{j}+\sum_{j=1}^{n} y_{j}\left(p_{a}-v_{a}\right)\right\} \\
=\frac{1}{p_{a}} \max _{\boldsymbol{x} \in X} \min _{\boldsymbol{y} \in Y}\left\{\sum_{j=1}^{n}\left(\sum_{i=1}^{m} a_{i j} x_{i}+p_{a}-v_{a}\right) y_{j}\right\} \\
=\frac{1}{p_{a}} \max _{\boldsymbol{x} \in X} \min _{j \in J}\left\{\sum_{i=1}^{m} a_{i j} x_{i}+p_{a}-v_{a}\right\}
\end{aligned}
$$

and

$$
\begin{aligned}
& \min _{\boldsymbol{x} \in X} \max _{\boldsymbol{y} \in Y}\left\{\boldsymbol{v}_{A}\left(\boldsymbol{x}^{\mathrm{T}} \boldsymbol{F} \boldsymbol{y}\right)\right\}=\min _{\boldsymbol{x} \in X} \max _{\boldsymbol{y} \in Y}\left\{\frac{v_{r}-\boldsymbol{x}^{\mathrm{T}} \boldsymbol{F} \boldsymbol{y}}{p_{r}}\right\} \\
& =\frac{1}{p_{r}} \min _{\boldsymbol{x} \in X} \max _{\boldsymbol{y} \in Y}\left\{v_{r}-\sum_{i=1}^{m} \sum_{j=1}^{n} a_{i j} x_{i} y_{j}\right\} \\
& =\frac{1}{p_{r}} \min _{\boldsymbol{x} \in X} \max _{\boldsymbol{y} \in Y}\left\{\sum_{j=1}^{n} v_{r} y_{j}-\sum_{i=1}^{m} \sum_{j=1}^{n} a_{i j} x_{i} y_{j}\right\} \\
& =\frac{1}{p_{r}} \min _{\boldsymbol{x} \in X} \max _{\boldsymbol{y} \in Y}\left\{\sum_{j=1}^{n} y_{j}\left(v_{r}-\sum_{i=1}^{m} a_{i j} x_{i}\right)\right\} \\
& =\frac{1}{p_{r}} \min _{x \in X} \max _{j \in J}\left\{v_{r}-\sum_{i=1}^{m} a_{i j} x_{i}\right\},
\end{aligned}
$$

respectively. Namely, Eq. (1) is simply rewritten as follows:

$$
\left\{\begin{array}{l}
\frac{1}{p_{a}} \max _{x \in X} \min _{j \in J}\left\{\sum_{i=1}^{m} a_{i j} x_{i}+p_{a}-v_{a}\right\} \\
\frac{1}{p_{r}} \min _{x \in X} \max _{j \in J}\left\{v_{r}-\sum_{i=1}^{m} a_{i j} x_{i}\right\}
\end{array}\right.
$$

Let

$$
\alpha=\min _{j \in J}\left\{\frac{1}{p_{a}}\left(\sum_{i=1}^{m} a_{i j} x_{i}+p_{a}-v_{a}\right)\right\}
$$

and

$$
\beta=\max _{j \in J}\left\{\frac{1}{p_{r}}\left(v_{r}-\sum_{i=1}^{m} a_{i j} x_{i}\right)\right\} .
$$

Hence, it is derived from Eq. (14) that $\max \{\alpha\}, \min \{\beta\}$

$$
\text { s.t. }\left\{\begin{array}{l}
\sum_{i=1}^{m} a_{i j} x_{i}+p_{a}-v_{a} \geq p_{a} \alpha \quad(j=1,2, \cdots n) \\
\sum_{i=1}^{m} a_{i j} x_{i}-v_{r} \geq-p_{r} \beta \quad(j=1,2, \cdots n) \\
x_{1}+x_{2}+\cdots+x_{m}=1 \\
0 \leq \alpha \leq 1,0 \leq \beta \leq 1 \\
\alpha+\beta \leq 1 \\
x_{i} \geq 0(i=1,2, \cdots, m)
\end{array}\right.
$$

Obviously, Eq. (15) is a bi-objective linear programming model of $m+2$ decision variables $\alpha, \beta$ and $X_{i} \quad(i=1,2, \cdots, m)$. Using the weighted average method, Eq. (15) may be transformed into Eq. (11).

Similarly, player II's maximin-minimax solution of the matrix game with the IF-set goal can be solved.

Theorem 3. Assume that $\mu_{B}$ and $v_{B}$ for player II's IFset goal are the linear functions defined by Eqs. (9) and (10). Player II's maximin-minimax solution of matrix games with IF-set goals can be obtained through solving the linear programming as follows:

$$
\begin{aligned}
& \min \{\eta-\lambda\} \\
& \text { s.t. }\left\{\begin{array}{l}
\sum_{j=1}^{n} a_{i j} y_{j}-\omega_{a}-q_{a} \leq-q_{a} \lambda(i=1,2, \cdots m) \\
\sum_{j=1}^{n} a_{i j} y_{j}-\omega_{r} \leq q_{r} \eta(i=1,2, \cdots m) \\
y_{1}+y_{2}+\cdots+y_{n}=1 \\
0 \leq \lambda \leq 1,0 \leq \eta \leq 1 \\
\lambda+\eta \leq 1 \\
y_{j} \geq 0(j=1,2, \cdots, n)
\end{array}\right.
\end{aligned}
$$

Proof. According to Eqs. (9) and (10), Eq. (2) can be obtained as follows:

$$
\begin{aligned}
& \max _{\boldsymbol{y} \in Y} \min _{\boldsymbol{x} \in X}\left\{\mu_{B}\left(\boldsymbol{x}^{\mathrm{T}} \boldsymbol{F} \boldsymbol{y}\right)\right\}=\max _{\boldsymbol{y} \in Y} \min _{\boldsymbol{x} \in X}\left\{1-\frac{\boldsymbol{x}^{\mathrm{T}} \boldsymbol{F} \boldsymbol{y}-\omega_{a}}{q_{a}}\right\} \\
& =\frac{1}{q_{a}} \max _{\boldsymbol{y} \in Y} \min _{\boldsymbol{x} \in X}\left\{-\boldsymbol{x}^{\mathrm{T}} \boldsymbol{F} \boldsymbol{y}+\omega_{a}+q_{a}\right\} \\
& =\frac{1}{q_{a}} \max _{\boldsymbol{y} \in Y} \min _{\boldsymbol{x} \in X}\left\{-\sum_{i=1}^{m} \sum_{j=1}^{n} a_{i j} x_{i} y_{j}+\sum_{i=1}^{m}\left(\omega_{a}+q_{a}\right) x_{i}\right\} \\
& =\frac{1}{q_{a}} \max _{\boldsymbol{y} \in Y} \min _{\boldsymbol{x} \in X}\left\{\sum_{i=1}^{m}\left(-\sum_{j=1}^{n} a_{i j} y_{j}+\omega_{a}+q_{a}\right) x_{i}\right\} \\
& =\frac{1}{q_{a}} \max _{\boldsymbol{y} \in Y} \min _{i \in I}\left\{-\sum_{j=1}^{n} a_{i j} y_{j}+\omega_{a}+q_{a}\right\}
\end{aligned}
$$

and

$$
\begin{aligned}
& \min _{\boldsymbol{y} \in Y} \max _{\boldsymbol{x} \in X}\left\{\boldsymbol{v}_{B}\left(\boldsymbol{x}^{\mathrm{T}} \boldsymbol{F} \boldsymbol{y}\right)\right\}=\min _{\boldsymbol{y} \in Y} \max _{\boldsymbol{x} \in X}\left\{\frac{\boldsymbol{x}^{\mathrm{T}} \boldsymbol{F} \boldsymbol{y}-\omega_{\mathrm{r}}}{q_{r}}\right\} \\
& =\frac{1}{q_{r}} \min _{\boldsymbol{y} \in Y} \max _{\boldsymbol{x} \in X}\left\{\sum_{i=1}^{m} \sum_{j=1}^{n} a_{i j} x_{i} y_{j}-\omega_{r}\right\} \\
& =\frac{1}{q_{r}} \min _{\boldsymbol{y} \in Y} \max _{\boldsymbol{x} \in X}\left\{\sum_{i=1}^{m} \sum_{j=1}^{n} a_{i j} x_{i} y_{j}-\sum_{i=1}^{m} \omega_{r} x_{i}\right\} \\
& =\frac{1}{q_{r}} \min _{\boldsymbol{y} \in Y} \max _{\boldsymbol{x} \in X}\left\{\sum_{i=1}^{m}\left(\sum_{j=1}^{n} a_{i j} y_{j}-\omega_{r}\right) x_{i}\right\} \\
& =\frac{1}{q_{r}} \min _{\boldsymbol{y} \in Y} \max _{i \in I}\left\{\sum_{j=1}^{n} a_{i j} y_{j}-\omega_{r}\right\},
\end{aligned}
$$


respectively. Namely, Eq. (2) can be simply rewritten as follows:

$$
\left\{\begin{array}{l}
\frac{1}{q_{a}} \max _{y \in Y} \min _{i \in I}\left\{-\sum_{j=1}^{n} a_{i j} y_{j}+\omega_{a}+q_{a}\right\} \\
\frac{1}{q_{r}} \min _{y \in Y} \max _{i \in I}\left\{\sum_{j=1}^{n} a_{i j} y_{j}-\omega_{r}\right\}
\end{array}\right.
$$

Let

$$
\lambda=\min _{i \in I}\left\{\frac{1}{q_{a}}\left(-\sum_{j=1}^{n} a_{i j} y_{j}+\omega_{a}+q_{a}\right)\right\}
$$

and

$$
\eta=\max _{i \in I}\left\{\frac{1}{q_{r}}\left(\sum_{j=1}^{n} a_{i j} y_{j}-\omega_{r}\right)\right\} .
$$

Then, Eq. (19) can be transformed into the bi-objective programming model as follows:

$$
\begin{aligned}
& \max \{\lambda\}, \min \{\eta\} \\
& \text { s.t. }\left\{\begin{array}{l}
\sum_{j=1}^{n} a_{i j} y_{j}-\omega_{a}-q_{a} \leq-q_{a} \lambda(i=1,2, \cdots m) \\
\sum_{j=1}^{n} a_{i j} y_{j}-\omega_{r} \leq q_{r} \eta(i=1,2, \cdots m) \\
y_{1}+y_{2}+\cdots+y_{n}=1 \\
0 \leq \lambda \leq 1,0 \leq \eta \leq 1 \\
\lambda+\eta \leq 1 \\
y_{j} \geq 0(j=1,2, \cdots, n)
\end{array}\right.
\end{aligned}
$$

In a similar consideration to Eq. (15), Eq. (20) can be transformed into Eq. (16). Thus, we have proven Theorem 3.

From the above discussions, we observe that solving the matrix games with IF-set goals can be transformed into solving Eqs. (11) and (16). Namely, if $\left(x^{*}, \alpha^{*}, \beta^{*}\right)$ is an optimal solution to Eq. (11), then $x^{*}$ is a maximinminimax strategy for player I , $\alpha^{*}$ and $\beta^{*}$ are the least degree of satisfaction of attainment of the IF-set goal and the largest degree of dissatisfaction of attainment of the IF-set goal for player I, respectively. The degree of hesitation of attainment of the IF-set goal is equal to $1-\alpha^{*}-\beta^{*}$. Similarly, an optimal solution $\left(y^{*}, \lambda^{*}, \eta^{*}\right)$ to Eq. (16) can be interpreted.

The following Theorems show that if IF-set goals are reduced to F-set goals for players then Eqs. (11) and (16) can be reduced to the models given by Bector et $\mathrm{al}^{2}$.

Theorem 4. If the degree of membership $\mu_{A}\left(\boldsymbol{x}^{\mathrm{T}} \boldsymbol{F} \boldsymbol{y}\right)$ and the degree of non-membership $v_{A}\left(\boldsymbol{x}^{\mathrm{T}} \mathbf{F y}\right)$ for player I's IF-set goal defined by Eqs. (3) and (4) satisfy $\mu_{A}\left(\boldsymbol{x}^{\mathrm{T}} \boldsymbol{F y}\right)+v_{A}\left(\boldsymbol{x}^{\mathrm{T}} \boldsymbol{F} y\right)=1$, i.e., the IF-set goal is reduced to a F-set goal, then I's maximin-minimax solution of the matrix game with the IF-set goal can be obtained through solving the linear programming as follows:

$$
\begin{aligned}
& \max \{u\} \\
& \text { s.t. }\left\{\begin{array}{l}
\sum_{i=1}^{m} a_{i j} x_{i}-v_{a} \geq p_{a}(u-1) \quad(j=1,2, \cdots n) \\
x_{1}+x_{2}+\cdots+x_{m}=1 \\
0 \leq u \leq 1 \\
x_{i} \geq 0(i=1,2, \cdots, m)
\end{array}\right.
\end{aligned}
$$

Proof. According to Eq. (3) and $\mu_{A}\left(\boldsymbol{x}^{\mathrm{T}} \boldsymbol{F} \boldsymbol{y}\right)+v_{A}\left(\boldsymbol{x}^{\mathrm{T}} \boldsymbol{F} \boldsymbol{y}\right)=1$, the degree of non-membership $v_{A}\left(\boldsymbol{x}^{\mathrm{T}} \boldsymbol{F} \boldsymbol{y}\right)$ for the player I's IF-set goal can be obtained as follows:

$$
v_{A}\left(\boldsymbol{x}^{\mathrm{T}} \boldsymbol{F} \boldsymbol{y}\right)=\frac{v_{a}-\boldsymbol{x}^{\mathrm{T}} \boldsymbol{F} \boldsymbol{y}}{p_{a}}
$$

Using Eqs. (3) and (22), Eq. (1) are calculated as follows:

$$
\begin{gathered}
\max _{\boldsymbol{x} \in X} \min _{\boldsymbol{y} \in Y}\left\{\mu_{A}\left(\boldsymbol{x}^{\mathrm{T}} \boldsymbol{F} \boldsymbol{y}\right)\right\}=\max _{\boldsymbol{x} \in X} \min _{\boldsymbol{y} \in Y}\left\{1-\frac{v_{a}-\boldsymbol{x}^{\mathrm{T}} \boldsymbol{F y}}{p_{a}}\right\} \\
=\frac{1}{p_{a}} \max _{\boldsymbol{x} \in X} \min _{\boldsymbol{y} \in Y}\left\{\sum_{i=1}^{m} \sum_{j=1}^{n} a_{i j} x_{i} y_{j}+p_{a}-v_{a}\right\} \\
=\frac{1}{p_{a}} \max _{\boldsymbol{x} \in X} \min _{\boldsymbol{y} \in Y}\left\{\sum_{i=1}^{m} \sum_{j=1}^{n} a_{i j} x_{i} y_{j}+\sum_{j=1}^{n} y_{j}\left(p_{a}-v_{a}\right)\right\} \\
=\frac{1}{p_{a}} \max _{x \in X} \min _{\boldsymbol{y} \in Y}\left\{\sum_{j=1}^{n}\left(\sum_{i=1}^{m} a_{i j} x_{i}+p_{a}-v_{a}\right) y_{j}\right\} \\
=\frac{1}{p_{a}} \max _{x \in X} \min _{j \in J}\left\{\sum_{i=1}^{m} a_{i j} x_{i}+p_{a}-v_{a}\right\}
\end{gathered}
$$

and

$$
\begin{aligned}
& \min _{\boldsymbol{x} \in X} \max _{\boldsymbol{y} \in Y}\left\{v_{A}\left(\boldsymbol{x}^{\mathrm{T}} \boldsymbol{F y}\right)\right\}=\min _{\boldsymbol{x} \in X} \max _{\boldsymbol{y} \in Y}\left\{\frac{v_{a}-\boldsymbol{x}^{\mathrm{T}} \boldsymbol{F} \boldsymbol{y}}{p_{a}}\right\} \\
& =\frac{1}{p_{a}} \min _{\boldsymbol{x} \in X} \max _{\boldsymbol{y} \in Y}\left\{v_{a}-\sum_{i=1}^{m} \sum_{j=1}^{n} a_{i j} x_{i} y_{j}\right\} \\
& =\frac{1}{p_{a}} \min _{\boldsymbol{x} \in X} \max _{\boldsymbol{y} \in Y}\left\{\sum_{j=1}^{n} v_{a} y_{j}-\sum_{i=1}^{m} \sum_{j=1}^{n} a_{i j} x_{i} y_{j}\right\} \\
& =\frac{1}{p_{a}} \min _{\boldsymbol{x} \in X} \max _{\boldsymbol{y} \in Y}\left\{\sum_{j=1}^{n} y_{j}\left(v_{a}-\sum_{i=1}^{m} a_{i j} x_{i}\right)\right\} \\
& =\frac{1}{p_{a}} \min _{\boldsymbol{x} \in X} \max _{j \in J}\left\{v_{a}-\sum_{i=1}^{m} a_{i j} x_{i}\right\}
\end{aligned}
$$

respectively. Namely, Eq. (1) is simply rewritten as follows:

$$
\left\{\begin{array}{l}
\frac{1}{p_{a}} \max _{x \in X} \min _{j \in J}\left\{\sum_{i=1}^{m} a_{i j} x_{i}+p_{a}-v_{a}\right\} \\
\frac{1}{p_{a}} \min _{x \in X} \max _{j \in J}\left\{v_{a}-\sum_{i=1}^{m} a_{i j} x_{i}\right\}
\end{array}\right.
$$




$$
\alpha=\min _{j \in J}\left\{\frac{1}{p_{a}}\left(\sum_{i=1}^{m} a_{i j} x_{i}+p_{a}-v_{a}\right)\right\}
$$

and

$$
\beta=\max _{j \in J}\left\{\frac{1}{p_{a}}\left(v_{a}-\sum_{i=1}^{m} a_{i j} x_{i}\right)\right\} .
$$

Hence, it is derived from Eq. (25) that

$$
\begin{aligned}
& \max \{\alpha\}, \min \{\beta\} \\
& \text { s.t. }\left\{\begin{array}{l}
\sum_{i=1}^{m} a_{i j} x_{i}-v_{a} \geq p_{a}(\alpha-1) \quad(j=1,2, \cdots n) \\
\sum_{i=1}^{m} a_{i j} x_{i}-v_{a} \geq-p_{a} \beta \quad(j=1,2, \cdots n) \\
x_{1}+x_{2}+\cdots+x_{m}=1 \\
0 \leq \alpha \leq 1,0 \leq \beta \leq 1 \\
\alpha+\beta \leq 1 \\
x_{i} \geq 0(i=1,2, \cdots, m)
\end{array}\right.
\end{aligned}
$$

It is easily seen that $\min \{\beta\}$ is equivalent to $\max \{1-\beta\}$ since $0 \leq \beta \leq 1$.Thus, using the weighted average method, $\max \{\alpha\}$ and $\min \{\beta\}$ in Eq. (26) may be written as follows:

$$
\max \{(\alpha+1-\beta) / 2\}
$$

The constraint condition in Eq. (26) may be written as follows:

$$
\text { s.t. }\left\{\begin{array}{l}
\sum_{i=1}^{m} a_{i j} x_{i}-v_{a} \geq p_{a}[(\alpha+1-\beta) / 2-1](j=1,2, \cdots n) \\
x_{1}+x_{2}+\cdots+x_{m}=1 \\
0 \leq \alpha \leq 1,0 \leq \beta \leq 1 \\
\alpha+\beta \leq 1 \\
x_{i} \geq 0(i=1,2, \cdots, m)
\end{array}\right.
$$

Let

$$
u=\alpha+1-\beta
$$

Combining with Eqs. (27)-(29), the bi-objective programming model (i.e., Eq. (26)) can be transformed into Eq. (21).

Obviously, Eq. (21) is just the model (i.e., (FLP)) given by Bector et al. ${ }^{2}$

Theorem 5. If the degree of membership $\mu_{B}\left(\boldsymbol{x}^{\mathrm{T}} \mathbf{F y}\right)$ and the degree of non-membership $v_{B}\left(x^{\mathrm{T}} \mathbf{F y}\right)$ for player II's IF-set goal defined by Eqs. (9) and (10) satisfy $\mu_{B}\left(\boldsymbol{x}^{\mathrm{T}} \boldsymbol{F} \boldsymbol{y}\right)+v_{B}\left(\boldsymbol{x}^{\mathrm{T}} \boldsymbol{F} \boldsymbol{y}\right)=1$, i.e., the IF-set goal is reduced to a F-set goal, then player II's maximin-minimax solution of the matrix game with the IF-set goal can be obtained through solving the linear programming as follows:

$$
\begin{aligned}
& \max \{v\} \\
& \text { s.t. }\left\{\begin{array}{l}
\sum_{j=1}^{n} a_{i j} y_{j}-v_{a} \geq p_{a}(1-v) \quad(i=1,2, \cdots m) \\
y_{1}+y_{2}+\cdots+y_{n}=1 \\
0 \leq v \leq 1 \\
y_{j} \geq 0(j=1,2, \cdots, n)
\end{array}\right.
\end{aligned}
$$

Proof. According to Eq. (9) and $\mu_{B}\left(\boldsymbol{x}^{\mathrm{T}} \boldsymbol{F} \boldsymbol{y}\right)+v_{B}\left(\boldsymbol{x}^{\mathrm{T}} \boldsymbol{F} \boldsymbol{y}\right)=1$, the degree of nonmembership $v_{A}\left(\boldsymbol{x}^{\mathrm{T}} \boldsymbol{F} \boldsymbol{y}\right)$ for player II's IF-set goal can be obtained as follows:

$$
v_{B}\left(\boldsymbol{x}^{\mathrm{T}} \boldsymbol{F} \boldsymbol{y}\right)=\left(\boldsymbol{x}^{\mathrm{T}} \boldsymbol{F} \boldsymbol{y}-\omega_{a}\right) / \boldsymbol{q}_{a}
$$

Using Eqs. (9) and (31), Eq. (2) can be obtained as follows:

$$
\begin{aligned}
& \max _{\boldsymbol{y} \in Y} \min _{\boldsymbol{x} \in X}\left\{\mu_{B}\left(\boldsymbol{x}^{\mathrm{T}} \boldsymbol{F} \boldsymbol{y}\right)\right\}=\max _{\boldsymbol{y} \in Y} \min _{\boldsymbol{x} \in X}\left\{1-\frac{\boldsymbol{x}^{\mathrm{T}} \boldsymbol{F} \boldsymbol{y}-\omega_{a}}{q_{a}}\right\} \\
& =\frac{1}{q_{a}} \max _{\boldsymbol{y} \in Y} \min _{\boldsymbol{x} \in X}\left\{-\boldsymbol{x}^{\mathrm{T}} \boldsymbol{F} \boldsymbol{y}+\omega_{a}+q_{a}\right\} \\
& =\frac{1}{q_{a}} \max _{\boldsymbol{y} \in Y} \min _{\boldsymbol{x} \in X}\left\{-\sum_{i=1}^{m} \sum_{j=1}^{n} a_{i j} x_{i} y_{j}+\sum_{i=1}^{m}\left(\omega_{a}+q_{a}\right) x_{i}\right\} \\
& =\frac{1}{q_{a}} \max _{\boldsymbol{y} \in Y} \min _{\boldsymbol{x} \in X}\left\{\sum_{i=1}^{m}\left(-\sum_{j=1}^{n} a_{i j} y_{j}+\omega_{a}+q_{a}\right) x_{i}\right\} \\
& =\frac{1}{q_{a}} \max _{\boldsymbol{y} \in Y} \min _{i \in I}\left\{-\sum_{j=1}^{n} a_{i j} y_{j}+\omega_{a}+q_{a}\right\}
\end{aligned}
$$

and

$$
\begin{aligned}
& \min _{\boldsymbol{y} \in Y} \max _{\boldsymbol{x} \in X}\left\{v_{B}\left(\boldsymbol{x}^{\mathrm{T}} \boldsymbol{F} \boldsymbol{y}\right)\right\}=\min _{\boldsymbol{y} \in Y} \max _{\boldsymbol{x} \in X}\left\{\frac{\boldsymbol{x}^{\mathrm{T}} \boldsymbol{F} \boldsymbol{y}-\omega_{a}}{q_{a}}\right\} \\
& =\frac{1}{q_{a}} \min _{\boldsymbol{y} \in Y} \max _{\boldsymbol{x} \in X}\left\{\sum_{i=1}^{m} \sum_{j=1}^{n} a_{i j} x_{i} y_{j}-\omega_{a}\right\} \\
& =\frac{1}{q_{a}} \min _{\boldsymbol{y} \in Y} \max _{\boldsymbol{x} \in X}\left\{\sum_{i=1}^{m} \sum_{j=1}^{n} a_{i j} x_{i} y_{j}-\sum_{i=1}^{m} \omega_{a} x_{i}\right\} \\
& =\frac{1}{q_{a}} \min _{\boldsymbol{y} \in Y} \max _{\boldsymbol{x} \in X}\left\{\sum_{i=1}^{m}\left(\sum_{j=1}^{n} a_{i j} y_{j}-\omega_{a}\right) x_{i}\right\} \\
& =\frac{1}{q_{a}} \min _{\boldsymbol{y} \in Y} \max _{i \in I}\left\{\sum_{j=1}^{n} a_{i j} y_{j}-\omega_{a}\right\},
\end{aligned}
$$

respectively. Namely, Eq. (2) can be simply rewritten as follows:

$$
\left\{\begin{array}{l}
\frac{1}{q_{a}} \max _{y \in Y} \min _{i \in I}\left\{-\sum_{j=1}^{n} a_{i j} y_{j}+\omega_{a}+q_{a}\right\} \\
\frac{1}{q_{a}} \min _{\boldsymbol{y} \in Y} \max _{i \in I}\left\{\sum_{j=1}^{n} a_{i j} y_{j}-\omega_{a}\right\}
\end{array}\right.
$$

Let

$$
\lambda=\min _{i \in I}\left\{\frac{1}{q_{a}}\left(-\sum_{j=1}^{n} a_{i j} y_{j}+\omega_{a}+q_{a}\right)\right\}
$$

and 


$$
\eta=\max _{i \in I}\left\{\frac{1}{q_{a}}\left(\sum_{j=1}^{n} a_{i j} y_{j}-\omega_{a}\right)\right\} .
$$

Then, Eq. (34) can be transformed into the bi-objective programming model as follows:

$$
\begin{aligned}
& \max \{\lambda\}, \min \{\eta\} \\
& \text { s.t. }\left\{\begin{array}{l}
\sum_{j=1}^{n} a_{i j} y_{j}-\omega_{a}-q_{a} \leq-q_{a} \lambda \\
\sum_{j=1}^{n} a_{i j} y_{j}-\omega_{a} \leq q_{a} \eta \\
y_{1}+y_{2}+\cdots+y_{n}=1 \\
0 \leq \lambda \leq 1,0 \leq \eta \leq 1 \\
\lambda+\eta \leq 1 \\
y_{j} \geq 0(j=1,2, \cdots, n)
\end{array}\right.
\end{aligned}
$$

It is easily seen that $\min \{\eta\}$ is equivalent to $\max \{1-\eta\}$ since $0 \leq \eta \leq 1$. Thus, using the weighted average method, $\max \{\lambda\}$ and $\min \{\eta\}$ in Eq. (35) may be written as follows:

$$
\max \{(\lambda+1-\eta) / 2\}
$$

The constraint condition in Eq. (35) may be written as follows:

$$
\text { s.t. }\left\{\begin{array}{l}
\sum_{j=1}^{n} a_{i j} y_{j}-v_{a} \geq p_{a}[(1-\lambda+\eta) / 2] \quad(i=1,2, \cdots m) \\
y_{1}+y_{2}+\cdots+y_{n}=1 \\
0 \leq \lambda \leq 1,0 \leq \eta \leq 1 \\
\lambda+\eta \leq 1 \\
y_{j} \geq 0(j=1,2, \cdots, n)
\end{array}\right.
$$

Due to the fact $(1-\lambda+\eta) / 2=1-(\lambda+1-\eta) / 2$, Eq. (37) can be further rewritten as follows:

$$
\text { s.t. }\left\{\begin{array}{l}
\sum_{j=1}^{n} a_{i j} y_{j}-v_{a} \geq p_{a}[1-(\lambda+1-\eta) / 2](i=1,2, \cdots m) \\
y_{1}+y_{2}+\cdots+y_{n}=1 \\
0 \leq \lambda \leq 1,0 \leq \eta \leq 1 \\
\lambda+\eta \leq 1 \\
y_{j} \geq 0(j=1,2, \cdots, n)
\end{array}\right.
$$

Let

$$
v=(\lambda+1-\eta) / 2
$$

Combining with Eqs. (36), (38) and (39), the biobjective programming model (i.e., Eq. (35)) can be transformed into Eq. (30).

Obviously, Eq. (30) is just the model (i.e., (FLD)) given by Bector et al. ${ }^{2}$
Theorems 4 and 5 show that the proposed models in this paper are the generalization of those given by Bector et al. ${ }^{2}$ It is easily seen that using Eqs. (11) and (16), the least degrees of satisfaction of attainment of the IF-set goals and the largest degrees of dissatisfaction of attainment of the IF-set goals for players are obtained, as well as the degrees of hesitation of attainment of the IF-set goals for players. However, according to the models (i.e., Eqs. (21) and (30)) given by Bector et al. ${ }^{2}$, only the least degrees of satisfaction of attainment of the F-set goals are obtained and the degrees of dissatisfaction of attainment of the F-set goals are automatically equal to the compliments to 1 , i.e., the degrees of hesitation of attainment of the F-set goals for players are always equal to zero.

\section{An application to the market share problem}

Suppose that there are two companies $C_{1}$ and $C_{2}$ aiming to enhance the sales amount and market share of a product in a targeted market. $C_{1}$ has two pure strategies $\delta_{1}$ and $\delta_{2} . C_{2}$ has three pure strategies $\sigma_{1}$, $\sigma_{2}$ and $\sigma_{3}$. Under these strategies, the payoff matrix $\boldsymbol{F}$ is given as follows:

$$
\boldsymbol{F}=\frac{\sigma_{1}}{\delta_{1}}\left(\begin{array}{ccc}
4 & \sigma_{2} & \sigma_{3} \\
\delta_{2} & -1 \\
-2 & 0 & 1
\end{array}\right) .
$$

The aspiration level and the corresponding tolerance error of $C_{1}$ are designated as $v_{a}=3$ and $p_{a}=4$, respectively. The rejection level and the corresponding tolerance error of $C_{1}$ are designated as $v_{r}=2$ and $p_{r}=6$, respectively. The aspiration level and the corresponding tolerance error of $C_{2}$ are $\omega_{a}=-2$ and $q_{a}=5$, respectively. The rejection level and the corresponding tolerance error of $C_{2}$ are $\omega_{r}=0$ and $q_{r}=4$, respectively.

According to Eq. (11), the linear programming is constructed as follows:

$$
\begin{aligned}
& \max \{\alpha-\beta\} \\
& \text { s.t. }\left\{\begin{array}{l}
4 x_{1}-2 x_{2}+2 \geq 4 \alpha \\
2 x_{1}+2 \geq 4 \alpha \\
-x_{1}+x_{2}+2 \geq 4 \alpha \\
4 x_{1}-2 x_{2}-2 \geq-6 \beta \\
2 x_{1}-2 \geq-6 \beta \\
-x_{1}+x_{2}-2 \geq-6 \beta \\
x_{1}+x_{2}=1 \\
0 \leq \alpha \leq 1,0 \leq \beta \leq 1 \\
\alpha+\beta \leq 1 \\
x_{1} \geq 0, x_{2} \geq 0
\end{array}\right.
\end{aligned}
$$


Solving Eq. (40) using the Simplex method for the linear programming, an optimal solution $\left(x^{*}, \alpha^{*}, \beta^{*}\right)$ can be obtained, where $\boldsymbol{x}^{*}=(0.375,0.625)^{\mathrm{T}}, \quad \alpha^{*}=0.563$ and $\beta^{*}=0.292$, respectively.

The maximin-minimax strategy of $C_{1}$ is $\boldsymbol{x}^{*}=(0.375,0.625)^{\mathrm{T}}$, i.e., $x_{1}=0.375$ and $x_{2}=0.625$. In this case, the least degree of satisfaction of attainment of the IF-set goal and the largest degree of dissatisfaction of attainment of the IF-set goal for $C_{1}$ are 0.563 and 0.292 , respectively. The degree of hesitation of attainment of the IF-set goal is 0.145 .

Similarly, according to Eq. (16), the linear programming model is constructed as follows:

$$
\text { s.t. }\left\{\begin{array}{l}
4 y_{1}+2 y_{2}-y_{3}-3 \leq-5 \lambda \\
-2 y_{1}+y_{3}-3 \leq-5 \lambda \\
4 y_{1}+2 y_{2}-y_{3} \leq 4 \eta \\
-2 y_{1}+y_{3} \leq 4 \eta \\
y_{1}+y_{2}+y_{3}=1 \\
0 \leq \lambda \leq 1,0 \leq \eta \leq 1 \\
\lambda+\eta \leq 1 \\
y_{1} \geq 0, y_{2} \geq 0, y_{3} \geq 0
\end{array}\right.
$$

The optimal solution $\left(\boldsymbol{y}^{*}, \eta^{*}, \lambda^{*}\right)$ can be obtained, where $y^{*}=(0.25,0,0.75)^{\mathrm{T}}, \lambda^{*}=0.550$ and $\eta^{*}=0.063$, respectively. Thus, the maximin-minimax strategy of $C_{2}$ is $\boldsymbol{y}^{*}=(0.25,0,0.75)^{\mathrm{T}}$, i.e., $y_{1}=0.25, \quad y_{2}=0$, $y_{3}=0.75$. The least degree of satisfaction of attainment of the IF-set goal and the largest degree of dissatisfaction of attainment of the IF-set goal for $C_{2}$ are 0.550 and 0.063 , respectively. The degree of hesitation of attainment of the IF-set goal is 0.387 .

If the expected goal of the company $C_{1}$ is expressed with the F-set rather than the IF-set, i.e., the company $C_{1}$ has a F-set goal, then according to Eq. (21), the linear programming model can be constructed as follows:

$$
\begin{aligned}
& \max \{u\} \\
& \text { s.t. }\left\{\begin{array}{l}
4 x_{1}-2 x_{2}+2 \geq 4 u \\
2 x_{1}+2 \geq 4 u \\
-x_{1}+x_{2}+2 \geq 4 u \\
x_{1}+x_{2}=1 \\
0 \leq u \leq 1 \\
x_{1} \geq 0, x_{2} \geq 0
\end{array}\right.
\end{aligned}
$$

Solving Eq. (42), an optimal solution $\left(\boldsymbol{x}^{*}, u^{*}\right)$ can be obtained, where $x^{*}=(0.375,0.625)^{T}$ and $u^{*}=0.563$. Thus, the maximin strategy of $C_{1}$ is $x^{*}=(0.375,0.625)^{\mathrm{T}}$, i.e., $x_{1}=0.375$ and $x_{2}=0.625$. The least degree of satisfaction of attainment of the fuzzy goal for $C_{1}$ is 0.563 .

Similarly, if the company $C_{2}$ has a F-set goal, then according to Eq. (30), the linear programming model is constructed as follows:

$$
\begin{aligned}
& \max \{v\} \\
& \text { s.t. }\left\{\begin{array}{l}
4 y_{1}+2 y_{2}-y_{3}-3 \leq-5 v \\
-2 y_{1}+y_{3}-3 \leq-5 v \\
y_{1}+y_{2}+y_{3}=1 \\
0 \leq v \leq 1 \\
y_{1} \geq 0, y_{2} \geq 0, y_{3} \geq 0
\end{array}\right.
\end{aligned}
$$

Solving Eq. (43), an optimal solution $\left(\boldsymbol{y}^{*}, v^{*}\right)$ can be obtained, where $y^{*}=(0.25,0,0.75)^{\mathrm{T}}$ and $v^{*}=0.550$. Thus, the maximin strategy of $C_{2}$ is $y^{*}=(0.25,0,0.75)^{\mathrm{T}}$, i.e., $y_{1}=0.25, y_{2}=0$ and $y_{3}=0.75$. The least degree of satisfaction of attainment of the fuzzy goal for $C_{2}$ is 0.550 .

By comparison analysis, it is not difficult to see that using the proposed models in this paper and the models (i.e., Eqs. (21) and (30)) given by Bector et al ${ }^{2}$, we can obtain the strategies and the least degrees of satisfaction of attainment of the IF-set (or F-set) goals for the companies $C_{1}$ and $C_{2}$. However, using the proposed models in this paper, we can also obtain the largest degrees of dissatisfaction and the degrees of hesitation of attainment of the IF-set goals for the companies $C_{1}$ and $C_{2}$, which are of importance for $C_{1}$ and $C_{2}$. For example, using Eq. (43), the least degree of satisfaction of attainment of the F-set goal for $C_{2}$ is 0.550 , whereas the degree of dissatisfaction of attainment of the F-set goal for $C_{2}$ is automatically equal to $1-0.55$, i.e., 0.45 according to the Definition of the F-set. In this case, intuitively the company $C_{2}$ may not be sure whether the strategy $y^{*}=(0.25,0,0.75)^{\mathrm{T}}$ is optimal or not in that the degree of satisfaction and the degree of dissatisfaction of attainment of the F-set goal are approximately equal, especially when they are both equal to 0.5. However, using the proposed models in this paper, the largest degree of dissatisfaction of attainment of the IF-set goal for $C_{2}$ is only 0.063 , which may make $C_{2}$ believe that the strategy $y^{*}=(0.25,0,0.75)^{\mathrm{T}}$ is reasonable and 
reliable. The results show that the proposed models in this paper are reasonable and may provide more abundant information for players to make decision.

\section{Conclusions}

In this paper, we formulate the matrix games with IF-set goals and hereby develop the linear programming method, which is different from those proposed by $\mathrm{Li}$ and $\mathrm{Nan}^{27}$ and $\mathrm{Li}^{28}$. On the one hand, the discussed matrix games are remarkably different. The matrix games discussed in this paper are ones in which uncertainty only appears in goals of players, i.e., the goals of players are expressed with the IF-sets whereas payoffs of players are real numbers rather than the IFsets. The matrix games discussed in $\mathrm{Li}$ and $\mathrm{Nan}^{27}$ and $\mathrm{Li}^{28}$ are ones in which uncertainty only appears in payoffs of players, i.e., the payoffs of players are expressed with the IF-sets whereas goals of players are not taken into consideration. On the other hand, the constructed bi-objective programming models and hereby the derived auxiliary non-linear/linear programming models are remarkably different. The main reason is that in this paper both the membership and non-membership functions of IF-set goals for players are optimized simultaneously whereas in $\mathrm{Li}$ and $\mathrm{Nan}^{27}$ and $\mathrm{Li}^{28}$ the expected payoff of player $\mathrm{I}$ is optimized according to the inclusion relation on the IFsets $^{16,17}$. Moreover, the proposed models in this paper are a natural generalization of those developed by Bector et $\mathrm{al}^{2}$. It is easily seen that the proposed models in this paper may provide more information than those ${ }^{2}$. Namely, the proposed models in this paper provide not only the least degree of satisfaction of attainment of the IF-set goals but also the largest degree of dissatisfaction and the degree of hesitation of attainment of the IF-set goals. The results show that the IF-set may express information more abundant and flexible than the F-set when it is used to deal with uncertainty in game theory. Naturally, in many cases both goals and payoffs of players may be the IF-sets. Such a kind of matrix games will be investigated in the future.

\section{Acknowledgements}

This research was supported by the key program of National Natural Science Foundation of China (No. 71231003), the Natural Science Foundation of China (Nos. 71171055, 71101033 and 71001015), the Program for New Century Excellent Talents in University (the
Ministry of Education of China, NCET-10-0020), the Specialized Research Fund for the Doctoral Program of Higher Education of China (No. 20113514110009), the Natural Science foundation for Distinguished Young Scholars of Guangxi (No. 2012GXNSFFA060003) and the Natural Science of Guangxi (No. 2012GXNSFAA053013).

\section{References}

1. C. R. Bector and S. Chandra, Fuzzy Mathematical Programming and Fuzzy Matrix Games (Springer Verlag, Berlin, Germany, 2005).

2. C. R. Bector, S. Chandra and V. Vijay, Matrix games with fuzzy goals and fuzzy linear programming duality, Fuzzy Optimization and Decision Making 3 (2004) 255269.

3. C. R. Bector, S. Chandra and V. Vijay, Duality in linear programming with fuzzy parameters and matrix games with fuzzy pay-offs, Fuzzy Sets and Systems 46 (2) (2004) 253-269.

4. L. Campos, Fuzzy linear programming models to solve fuzzy matrix games, Fuzzy Sets and Systems 32 (1989) 275-289.

5. L. Campos and A. Gonzalez, Fuzzy matrix games considering the criteria of the players, Kybernetes $\mathbf{2 0}$ (1991) 17-23.

6. L. Campos, A. Gonzalez and M. A. Vila, On the use of the ranking function approach to solve fuzzy matrix games in a direct way, Fuzzy Sets and Systems 49 (1992) 193-203.

7. D. Dubois and H. Prade, Fuzzy Sets and Systems Theory and Applications (Academic Press, New York, 1980).

8. D. F. Li, Fuzzy Multiobjective Many Person Decision Makings and Games (National Defense Industry Press, Beijing, 2003) pp. 196-326.

9. D. -F. Li, Fuzzy constrained matrix games with fuzzy payoffs, The Journal of Fuzzy Mathematics 7(4) (1999) 873-880.

10. D. -F. Li, A fuzzy multiobjective programming approach to solve fuzzy matrix games, The Journal of Fuzzy Mathematics 7 (4) (1999) 907-912.

11. S. T. Liu and C. Kao, Solution of fuzzy matrix games: an application of the extension principle, International journal of intelligent systems 22 (2007) 891-903.

12. I. Nishizaki and M. Sakawa, Equilibrium solutions in multiobjective bimatrix games with fuzzy payoffs and fuzzy goals, Fuzzy Sets and Systems 115 (2000) 105119.

13. I. Nishizaki and M. Sakawa, Fuzzy and Multiobjective Games for Conflict Resolution (Physica-Verlag, Heidelberg, 2001).

14. V. Vijay, S. Chandra and C. R. Bector, Matrix games with fuzzy goals and fuzzy payoffs, Omega: The International Journal of Management 33 (2005) 425-429. 
15. V. Vijay, A. Mehra, S. Chandra, and C.R. Bector, Fuzzy matrix games via a fuzzy relation approach, Fuzzy Optimization and Decision Making 6 (2007) 299-314.

16. K. T. Atanassov. Intuitionistic fuzzy sets, Fuzzy Sets and Systems 50 (1986) 87-96.

17. K. T. Atanassov, Intuitionistic Fuzzy Set (SpringerVerlag, Heidelberg, 1999).

18. K. T. Atanassov. Two theorems for intuitionistic fuzzy sets, Fuzzy Sets and Systems 110 (2000) 267-269.

19. S. K. De, R. Biswas and A. R. Roy, An application of intuitionistic fuzzy sets in medical diagnosis, Fuzzy Sets and Systems 117(2001) 209-213.

20. D. -F. Li and C. T. Cheng, New similarity measures of intuitionistic fuzzy sets and application to pattern recognitions, Pattern Recognition Letters 23 (2002) 221225.

21. D. -F. Li, Multiattribute decision making models and methods using intuitionistic fuzzy sets, Journal of Computer and System Sciences 70 (2005) 73-85.

22. H. W. Liu and G. J.Wang, Multi-criteria decision-making methods based on intuitionistic fuzzy sets, European Journal of Operational Research 179 (2007) 220-233.

23. K. T. Atanassov, Ideas for intuitionistic fuzzy equations, inequalities and optimization, Notes on Intuitionistic Fuzzy Sets 1 (1) (1995) 17-24.

24. D. Dimitrov, Market Structure: An Intuitionistic Fuzzy Approach (Economy University Publishing House, Sofia, 2000).

25. D. Dimitrov, On intuitionistic fuzzy consent rules, Notes on Intuitionistic Fuzzy Sets 7 (4) (2001) 65-69.

26. K. I. Tenekedjiev, N. D. Nikolova, C. A. Kobashikawa, K. Hirota. Conservative betting on sport games with intuitionistic fuzzy described uncertainty. 3rd IEEE International Conference on Intelligent Systems, Sept. 46, 2006, London, England, pp. 733-740.

27. D. -F. Li and J. -X. Nan, A nonlinear programming approach to matrix games with payoffs of Atanassov's intuitionistic fuzzy sets, International Journal of Uncertainty, Fuzziness and Knowledge-Based Systems 17 (4) (2009) 585-607.

28. D. -F. Li, Mathematical programming approach to matrix games with payoffs represented by Atanassov's interval valued intuitionistic fuzzy sets, IEEE Transactions on Fuzzy Systems 18 (6) (2010) 1112-1128. 